\title{
Valoración de la calidad del servicio educativo en instituciones educativas privadas
}

Rosa Katherine Cueto Huayascachi ${ }^{1}$ Luis Alberto Geraldo CAmpos ${ }^{2}$ Pedro Leonardo Tito Huamaní ${ }^{3}$

Recibido: 20/12/2019 Aceptado: 01/06/2020 Publicado: 16/10/2020

\begin{abstract}
RESUMEN
El objetivo de la presente investigación fue evaluar la calidad del servicio educativo en las instituciones educativas privadas del distrito de Chaclacayo, Lima. El estudio se realizó bajo la metodología cuantitativa de tipo descriptivo. Se evaluó la calidad del servicio educativo en 5 instituciones privadas de nivel secundaria, con una muestra de 209 participantes, entre alumnos, docentes y padres de familia; se aplicaron 3 cuestionarios, el primero, de 108 preguntas para estudiantes, el segundo, de 121 preguntas para docentes, y el tercero, de 83 preguntas para padres de familia. Los resultados de la investigación mostraron, en lo referido a la calidad del servicio educativo, que dichas instituciones se encuentran en el nivel "medio", según los siguientes criterios evaluados: satisfacción al cliente $(50.23 \%)$, liderazgo educativo $(51.37 \%)$, planeación estratégica aplicada a la educación (48.30\%), satisfacción del talento humano $(51.67 \%)$, gestión del talento humano (48.15\%), procesos educativos $(51.13 \%)$, impacto en la sociedad $(52.80 \%)$, resultados de la institución educativa $(50.17 \%)$ y recursos $(47.93 \%)$. En conclusión, la calidad del servicio educativo en el nivel secundario y en la categoría de colegios particulares en el distrito de Chaclacayo se encuentra en un nivel medio, representado por $50.93 \%$, según el promedio de percepciones del cliente externo (alumnos y padres de familia), como de los clientes internos (docentes).
\end{abstract}

Palabras clave: calidad total; educación; satisfacción; cliente; liderazgo; gestión; servicio; talento.

\section{INTRODUCCIÓN}

La preocupación por la mejora de la educación es cada vez mayor, dado que, en la historia, el desarrollo de las sociedades está sustentado en la mejor y mayor cualificación de sus miembros. En América Latina, la calidad de los servicios educativos pasa por un proceso de estancamiento, debido a que los decisores de las políticas macroeconómicas en la mayoría de los países privilegian otros indicadores, como la economía, la producción o el turismo, mas no una educación de calidad (Sánchez, 11 de abril de 2015). En este contexto, la Organización de las Naciones Unidas para la Educación, la Ciencia y la Cultura (UNESCO) menciona que la educación en América Latina y el Caribe se encuentra en franca decadencia, pues existen por lo menos 73 millones de personas que apenas saben leer, contar y escribir para subsistir, lo que representa un gran problema para el desarrollo de los países comprendidos en esta región (Barón, 31 de octubre de 2016).

La Organización para la Cooperación y el Desarrollo Económicos (OCDE) mide periódicamente el estado de la educación a nivel mundial y lo hace a través de la prueba PISA. Esta es el indicador más completo y, además, una herramienta que los países y las economías pueden usar para medir la calidad educativa desde una perspectiva internacional (Organization for Economic Cooperation and Development, 2019). De acuerdo a los últimos resultados de la prueba PISA, el Perú destaca al ocupar el puesto 64 de 77 países, lo que revela la mejora en comprensión lectora con un promedio de 401 , en matemática con un promedio de 400 y en ciencias con un promedio de 404 . Deberá mencionarse que estos resultados nos ponen por debajo de Argentina

1 Licenciada en Administración y Negocios Internacionales por la Universidad Peruana Unión (Lima, Perú). Actualmente, es asistente de importaciones en Import Tools. (Lima, Perú). ORCID: https://orcid.org/0000-0002-9237-9710

E-mail: rouseleo17@gmail.com

2 Licenciado en Administración con mención en Gestión Empresarial por la Universidad Peruana Unión (Lima, Perú). Actualmente, es docente investigador en la Escuela Profesional de Administración de la Universidad Peruana Unión. (Lima, Perú). ORCID: https://orcid.org/0000-0002-8366-689X E-mail: luis.geraldo@upeu.edu.pe

3 Doctor en Ciencias Administrativas por la Universidad Nacional Mayor de San Marcos (Lima, Perú). Actualmente, es docente principal e investigador en la misma universidad. (Lima, Perú). ORCID: https://orcid.org/0000-0002-2989-9203 E-mail: ptitoh@unmsm.edu.pe 
(Gestión, 3 de diciembre de 2019). Al respecto, el Centro de Desarrollo de la OCDE (2016) ya había afirmado que "Ios bajos resultados del país en las mediciones internacionales del aprendizaje siguen indicando que el gran reto por adelante es elevar la calidad de educación para todos los peruanos" (pp. 13-14).

Sin embargo, el Perú ha venido sumando esfuerzos, con un crecimiento económico promedio de $5.1 \%$ entre 2000 y 2015 , superior al $3.1 \%$ de América Latina y el Caribe en ese mismo periodo. Este crecimiento económico permitió al ciudadano promedio lograr mejor bienestar y mayor accesibilidad de oportunidades. A pesar de ello, el Perú sigue enfrentando grandes desafíos para convertirse en un país con desarrollo inclusivo y sostenible (Organización para la Cooperación y el Desarrollo Económicos, 2016; Organization for Economic Cooperation and Development, 2019). En esa dirección, también hay avances en el sistema educativo, como el mayor acceso de la población a la oferta educativa privada, así como el incremento de la tasa de matriculados a nivel primaria en $84 \%$ y en $78 \%$ a nivel secundario.

Asimismo, la inversión del Perú en educación ha aumentado, pero todavía dista de ser significativa. A nivel del producto bruto interno (PBI), el gasto en educación pasó de $2.9 \%$ en 2010 a $3.6 \%$ en 2015 ; sin embargo, la calidad educativa en nivel secundario aún se mantiene muy baja (Organización para la Cooperación y el Desarrollo Económicos, 2016). En ese orden de ideas, la Organización de las Naciones Unidas para la Educación, la Ciencia y la Cultura (2015) afirma que la educación se encuentra insuficientemente financiada, a pesar de incrementos presupuestales en muchos países. A raíz de esta necesidad, se creó un acuerdo que está plasmado en la declaración de "Educación para Todos", en concordancia con la Agenda 2030 promovida por la UNESCO. De acuerdo con esta organización, los Estados deben promover, como meta de inversión en educación, el $6 \%$ del PBI y/o el $20 \%$ de su presupuesto general. Para el caso peruano, el presupuesto en educación es de $3.6 \%$ del PBI, un porcentaje distante del que promueve el mencionado organismo (Robalino, 11 de julio de 2016).

Según Parodi (6 de diciembre de 2013), una educación de calidad genera buena rentabilidad, tanto para quien la recibe como para la sociedad misma. En efecto, "invertir en la calidad [...] reporta ventajas extraordinarias para la sociedad, [...] cómo mejorar la calidad de la educación es mucho menos evidente" (Hanushek, 2005, p. 15).
De la misma forma, incrementar y mejorar el capital humano permite un crecimiento económico sostenible. En tal sentido, los países necesitan trabajar con seres productivos y competentes, como resultado de recibir una educación de calidad (Vexler, 5 de octubre de 2016); lo contrario genera menores posibilidades de desarrollo en sus capacidades. Entonces, se trata de lograr un mejor aprovechamiento de oportunidades profesionales para desenvolverse exitosamente en la sociedad y su entorno (Villarreal, 2014). En consecuencia, la realidad del mercado laboral se refleja en que, de cada tres empresas, una no encuentra trabajadores con las competencias necesarias, debido a la baja formación educativa, que impide acceder a un empleo de calidad (Gonzales, 6 de abril de 2016).

En el contexto peruano, específicamente en la región Lima, existen 5552 colegios públicos y 11230 colegios privados. A nivel de estudiantes, aproximadamente un millón estudian en colegios públicos, y otro tanto, en los colegios privados (La República, 8 de enero de 2017). En los últimos veinte años, crecieron exponencialmente los colegios privados, institutos y universidades para atender la demanda. Sin embargo, si se considera la acreditación y certificación de calidad, según un informe de la consultora Proexpansión (2014), que valora la calidad educativa en los colegios de Lima Metropolitana, solo 200 de los 3862 colegios evaluados alcanzaron la certificación de calidad. Frente a la necesidad de determinar la calidad educativa ofertada por las instituciones, debemos ubicar en contexto lo descrito, a nivel de Lima en particular y a nivel del Perú en general. Es así que se toma como caso la evaluación de las instituciones privadas del distrito de Chaclacayo.

\section{METODOLOGÍA}

El enfoque corresponde a un estudio cuantitativo de corte descriptivo. Este alcance de estudio permite, por naturaleza, describir y especificar las propiedades y características importantes de la calidad del servicio en las instituciones educativas (IE) del sector privado en el distrito de Chaclacayo, en 2018. Además, corresponde a un diseño no experimental de tipo transversal descriptivo. Se trata de un diseño no experimental debido a que su naturaleza no pretende intervenir o manipular las variables de forma deliberada, pues solo se observaron los fenómenos en su contexto natural, para luego analizarlos. Además, es de naturaleza transversal porque la información se recolectó en un momento específico, es decir, en un tiempo único (Hernández-Sampieri, Fernández y Baptista, 2014). 


\section{Muestreo}

En un inicio, la población estuvo conformada por 15 instituciones educativas del sector privado, ubicadas en el distrito de Chaclacayo. La muestra fue de tipo no probabilístico, en la que priman los juicios del equipo investigador, tal como proponen Ñaupas, Mejía, Novoa y Villagómez (2014). Vale decir que las IE privadas, materia de la presente investigación, fueron las únicas que accedieron a colaborar para aplicar los instrumentos. Aquí, cabe señalar que se visitó al total de colegios ubicados en el distrito de Chaclacayo, se coordinó con sus respectivos directores o representantes y se les solicitó las facilidades para la aplicación de los tres instrumentos; pero, en el momento del trabajo de campo, solo 5 colegios del total aplicaron los instrumentos. Este hecho no resta seriedad a la investigación, cuyo desagregado se muestra en la Tabla 1.

Tabla 1. Distribución de la muestra.

\begin{tabular}{|l|c|}
\hline \multicolumn{1}{|c|}{ Grupo } & Muestra \\
\hline Estudiantes de $4 .^{\circ}$ y $5 .^{\circ}$ de secundaria & 101 \\
\hline Padres de familia & 79 \\
\hline Profesores & 29 \\
\hline Total & 209 \\
\hline
\end{tabular}

Fuente: Elaboración propia.

\section{Instrumentos y análisis de datos}

Para la presente investigación, se consideraron los 3 instrumentos elaborados por el psicopedagogo Héctor Mas (2014), que comprenden a estudiantes, docentes y padres de familia.

El primero, para estudiantes, agrupa 9 dimensiones (satisfacción del cliente, liderazgo, planeación estratégica, satisfacción del talento humano, gestión del talento humano, procesos educativos, impacto en la sociedad, resultados de la IE y recursos), con 108 ítems y una fiabilidad de alfa de Cronbach de 0.969 . Se caracteriza por ser de respuestas múltiples y utiliza la escala de Likert, donde 4 es igual a siempre y 1 es igual a nunca (Mas, 2014). En el caso de esta investigación, se obtuvo una fiabilidad de alfa de Cronbach de 0.973 (ver Tabla 2).

El segundo instrumento es para los docentes, agrupado en 9 dimensiones, con 121 ítems y con una fiabilidad de alfa de Cronbach de 0.962. Es de respuestas múltiples y utiliza la escala de Likert, donde 4 es igual a siempre y 1 es igual a nunca. Para la investigación, la fiabilidad de alfa de Cronbach fue de 0.989 (ver Tabla 2).
El tercer instrumento, dirigido a padres de familia, está conformado por 8 dimensiones (satisfacción del cliente, liderazgo, planeación estratégica, satisfacción del talento humano, procesos educativos, impacto en la sociedad, resultados de la IE y recursos), con 83 ítems y con una fiabilidad de alfa de Cronbach de 0.956. Para este caso, la fiabilidad de alfa de Cronbach fue de 0.979 (ver Tabla 2).

Tabla 2. Resumen de estadísticas de fiabilidad.

\begin{tabular}{|l|l|l|}
\hline \multicolumn{1}{|c|}{ Instrumentos } & \multicolumn{1}{|c|}{ Alfa de Cronbach } & \multicolumn{1}{c|}{$\mathbf{N} .^{\circ}$ de ítems } \\
\hline Alumnos & 0.973 & 108 \\
\hline Docentes & 0.989 & 121 \\
\hline Padres de familia & 0.979 & 83 \\
\hline
\end{tabular}

Fuente: Elaboración propia.

Posteriormente, se procedió a analizar los estadísticos descriptivos y de frecuencia de cada ítem, con lo que se obtuvieron los resultados según el instrumento aplicado. En seguida, se realizaron los cálculos de variables para proceder a la baremación de cada una de las dimensiones, con la finalidad de realizar el análisis de tablas cruzadas y, así, obtener los niveles de cada dimensión por instrumento. Finalmente, se realizaron los cálculos de dimensiones y sus respectivas baremaciones para determinar el nivel de calidad educativa, según percepciones de los alumnos, docentes y padres de familia.

\section{RESULTADOS}

Valoración de la calidad educativa según percepción de los estudiantes, docentes y padres de familia, por cada dimensión e institución educativa evaluada

En esta sección, se presenta un cuadro comparativo de todas las instituciones educativas evaluadas para conocer cuál de todas ellas posee un mejor nivel de satisfacción en las dimensiones de calidad educativa percibidas por los estudiantes, docentes y padres de familia.

La Tabla 3 permite revisar el nivel de cada dimensión por institución de acuerdo a los tres grupos de actores. En la IE Santísima Virgen de las Mercedes, la "satisfacción del cliente" revelada por los estudiantes fue del $30.4 \%$, seguida del $28.6 \%$ por los docentes y el $19.1 \%$ de satisfacción por parte de los padres de familia, a partir de una muestra de 60 encuestados de esta institución. En la IE Blas Pascal, los padres de familia se sienten satisfechos en un $51.1 \%$; los estudiantes, en un $21.7 \%$, y los docentes, en un $14.3 \%$, a partir de una muestra de 55 
encuestados en esta institución. Los resultados de la IE Eben Ezer, donde el $100 \%$ equivale a 51 personas encuestadas, el $21.7 \%$ de los estudiantes están satisfechos, así como el $21.4 \%$ de los docentes y el $6.4 \%$ de los padres de familia. Para el caso de la IE American School, se revela que del total de los encuestados, equivalente a 29 personas, el $17 \%$ de los padres de familia está satisfecho, seguido del $14.3 \%$ de los docentes y el $13 \%$ de los estudiantes. Finalmente, los resultados en cuanto a satisfacción en la IE Hans Christian Andersen muestran que el 21.4\% de los docentes están satisfechos, seguido del 13\% de los estudiantes y solo el $6.4 \%$ de los padres de familia, a partir de una muestra de 14 personas evaluadas en esta IE.

La dimensión "liderazgo educativo", evaluada en las 5 instituciones educativas, muestra una percepción dispersa, según los grupos de estudio. Así, en la IE Blas Pascal se refleja, para los padres de familia, un $49.1 \%$ de liderazgo; para los estudiantes, un $29.1 \%$, y para los docentes, solo $15 \%$. En el caso de la IE Eben Ezer, el 30\% de los docentes mencionó que esta tiene un liderazgo educativo, seguido del $27.3 \%$ revelado por los estudiantes y únicamente el $11.3 \%$ de parte de los padres de familia. Los resultados del liderazgo educativo en la IE Santísima Virgen de las Mercedes se vieron respaldados por el $25.5 \%$ de estudiantes, el $20 \%$ de los docentes y el $17 \%$ de los padres. Asimismo, para la IE American School, el $20 \%$ de los docentes, $15.1 \%$ de los padres y $14.5 \%$ de los estudiantes cree que la IE tiene liderazgo educativo. Por último, en la IE Hans Christian Andersen, el 15\% de los docentes menciona que su institución tiene liderazgo educativo, seguido del $7.5 \%$ de los padres y solo el $3.6 \%$ de los estudiantes.

La dimensión "planificación estratégica aplicada a la educación" también fue evaluada por los tres grupos de las 5 instituciones educativas. En ese sentido, la evaluación de la IE Santísima Virgen de las Mercedes reveló que el $37.3 \%$ de sus estudiantes percibe que existe planificación estratégica, el $20 \%$ de los docentes opina igual, así como el $15.8 \%$ de los padres de familia. Del mismo modo, en la IE Blas Pascal, el $50 \%$ de los padres de familia considera que existe planificación estratégica, al igual que el $26.7 \%$ de los docentes y el $17.6 \%$ de Ios estudiantes. En la IE Eben Ezer, del grupo de docentes encuestados, el $20 \%$ reveló que percibe planificación; de los estudiantes, el $17.6 \%$, y de los padres, solo un $10.5 \%$. Para la IE American School, del $100 \%$ de los encuestados, el $15.8 \%$ de padres reveló que percibe planificación en la IE, cifra similar al $15.7 \%$ de estudiantes y el $13.3 \%$ de padres.
Finalmente, en la IE Hans Christian Andersen, los resultados arrojaron que el $20 \%$ de los docentes señalan que hay planificación estratégica, mientras que solo el $7.9 \%$ de padres y el $5.9 \%$ de los estudiantes coinciden.

La dimensión "satisfacción del talento humano" en las instituciones educativas se aprecia de manera variada. Para el caso de la IE Blas Pascal, el 53.8\% de los padres percibe satisfacción en el talento humano, lo mismo que el $33.3 \%$ de los estudiantes y el $20 \%$ de los docentes. La encuesta en la IE Santísima Virgen de las Mercedes reveló que el $27.8 \%$ de los estudiantes, el $23.1 \%$ de los padres y el $20 \%$ de docentes creen que hay satisfacción en el talento humano de la IE. Para la IE Eben Ezer, el 20\% de los docentes muestra que existe satisfacción del talento humano, seguido por los estudiantes con el $19.4 \%$ y solo el $2.6 \%$ de los padres de familia. En cuanto a la IE American School, los resultados arrojaron que el $16.7 \%$ de los estudiantes, $13.3 \%$ de los docentes y solo el $10.3 \%$ de los padres piensa que existe satisfacción del talento humano en la IE. Finalmente, en la IE Hans Christian Andersen, el $26.7 \%$ de los docentes encuestados percibe satisfacción, mientras que solo el $10.3 \%$ de los padres y el $2.8 \%$ de los estudiantes también lo perciben.

En cuanto a la dimensión "gestión del talento humano", solo se aplicó a alumnos y docentes debido a que estos dos grupos son los que más tiempo pasan en la IE. De este modo, para el caso de la IE Santísima Virgen de las Mercedes, el 35\% de sus estudiantes perciben la existencia de gestión del talento humano, así como el $21.4 \%$ de los docentes. En la IE Blas Pascal, los resultados fueron menores, el $25 \%$ de los estudiantes ratifican la existencia de gestión del talento humano y únicamente el $14.3 \%$ de los docentes. En la IE American School, del $100 \%$ de los encuestados, el $28.6 \%$ de los docentes y el $17.5 \%$ de los estudiantes manifestó que existe gestión del talento humano en la institución. Sin embargo, en la IE Hans Christian Andersen solo el $21.4 \%$ de los docentes encuestados reveló que existe gestión del talento humano en la IE.

Los "procesos educativos" dentro de las instituciones son un elemento indispensable, por lo que se decidió medir está dimensión dentro de la valoración de la calidad educativa en las 5 instituciones. En tal sentido, en la IE Blas Pascal, el 53.3\% de los padres de familia reveló la existencia de procesos educativos, seguido del $29.1 \%$ de alumnos y el $17.6 \%$ de los docentes. En la IE Santísima Virgen de las Mercedes, el $27.3 \%$ de los estudiantes, seguido del $20 \%$ de los padres y el $17.6 \%$ de los docentes, piensa que existen procesos educativos. 
En el caso de la IE Eben Ezer, del total de los encuestados, el $25.5 \%$ de los estudiantes opina que existen procesos educativos; en cuanto a los docentes, el porcentaje fue de $17.6 \%$, y, con respecto a los padres, fue solo el $6.7 \%$. En la IE American School, los resultados arrojaron que el $25 \%$ de los docentes, el $15.6 \%$ de los padres y el $12.7 \%$ de los alumnos mencionan que existen procesos educativos. Por último, en la IE Hans Christian Andersen, el $23.5 \%$ de los docentes manifestó que en la IE existen procesos educativos, mientras que para los estudiantes y padres de familia fue de $5.5 \%$ y $4.4 \%$, respectivamente.

Otra de las dimensiones que cobra gran importancia para la valoración de una IE es el impacto que tiene en la sociedad, es por ello que se evaluó la dimensión "impacto en la sociedad", vista desde la perspectiva de los estudiantes, padres y docentes. En ese sentido, los resultados para la IE Blas Pascal arrojaron que el $59.5 \%$ de los padres, el $34.5 \%$ de los estudiantes y el $25 \%$ de los docentes manifestó que la IE tiene un impacto sustancial en la sociedad. En la IE Eben Ezer, los resultados muestran que el $27.6 \%$ de los alumnos y el $12.5 \%$ tanto de padres como de docentes mencionaron que la IE tiene impacto en la sociedad. Los resultados de la IE American School revelan que el $25 \%$ de los docentes, el $20.7 \%$ de los estudiantes y el $9.4 \%$ de los padres de familia manifiestan que la IE tiene impacto en la sociedad. Para el caso de la IE Santísima Virgen de las Mercedes, los resultados arrojaron que el $25 \%$ de los docentes, el $17.2 \%$ de los estudiantes y solo el $9.4 \%$ de los padres creen que la IE tiene impacto en la sociedad. Finalmente, en la IE Hans Christian Andersen, el $12.5 \%$ de los docentes y el $9.4 \%$ de los padres mencionan que la IE tiene un impacto en la sociedad.

La valoración de la calidad educativa también se ve reflejada en los resultados que la IE tenga, es por ello que es importante conocer los resultados de las instituciones evaluadas y si son de conocimiento y percepción de los grupos en estudio. En tal sentido, los resultados de la IE Santísima Virgen de las Mercedes revelan que el $29 \%$ de los estudiantes, el $21.4 \%$ de los docentes y solo el $2.3 \%$ de los padres manifiestan tener conocimiento de Ios resultados en la IE. Para la IE Eben Ezer, se evidencia que el $28.6 \%$ de los docentes, el $25.5 \%$ de los estudiantes y el $2.3 \%$ de los padres perciben que existen resultados en la IE. En cambio, los números de la IE Blas Pascal son alentadores, pues el $55.8 \%$ de los padres, $23.6 \%$ de los estudiantes y $14.3 \%$ de los docentes mencionan la existencia de resultados de la institución. En la IE American
School, los resultados arrojaron que el $18.6 \%$ de los padres, el $18.2 \%$ de los estudiantes y el $14.3 \%$ de los docentes conocen los resultados de la IE. Por último, para la IE Hans Christian Andersen, se revela que el $21.4 \%$ de los docentes, el $9.3 \%$ de los padres y solo el $3.6 \%$ de los estudiantes perciben resultados de la IE.

Finalmente, se consideró la dimensión "recursos" para averiguar si la IE cuenta con lo suficiente para dar un buen servicio. De este modo, en la IE Blas Pascal, las encuestas demostraron que el $42.9 \%$ de los padres, el $33.3 \%$ de los alumnos y solo el $9 \%$ de los docentes creen que la IE cuenta con recursos suficientes para proveer un buen servicio educativo. Para la IE Eben Ezer, los resultados arrojaron que el $29.6 \%$ de los estudiantes, el $27.3 \%$ de los docentes y el $14.3 \%$ de los padres mencionan que la IE posee los recursos necesarios. En cambio, para la IE American School, los resultados revelan que el $36.4 \%$ de los docentes, el $23.8 \%$ de los padres y el $22.2 \%$ de los alumnos mencionan que la IE cuenta con recursos suficientes. Los resultados en la IE Santísima Virgen de las Mercedes arrojaron que, para el $27.3 \%$ de los docentes, el $19 \%$ de los padres y el $14.8 \%$ de los estudiantes, la IE cuenta con suficientes recursos para brindar un buen servicio educativo. Cabe mencionar que los resultados en el ponderado global para la IE Hans Christian Andersen en esta dimensión son mínimos, por lo que la institución debe seguir trabajando para incrementar sus recursos y dar un buen servicio.

De esta forma, las instituciones educativas Blas Pascal y Santísima Virgen de las Mercedes son las instituciones que encabezan la lista, al contar con una buena calidad educativa. Es decir, para los estudiantes, docentes y padres de familia, estas dos instituciones son las que brindan mayor calidad educativa, por ende, son las que ofrecen mayor satisfacción (ver Tabla 3).

\section{Nivel de percepción por cada dimensión evaluada}

La Tabla 4 presenta el $x$. En el análisis global de las 5 IE privadas, los resultados arrojaron que el $50.5 \%$ de los estudiantes, el $51.9 \%$ de los padres de familia y el $48.3 \%$ de los docentes tienen un nivel de satisfacción medio y, en promedio, el $21.27 \%$ de los tres grupos tiene un nivel de satisfacción alto.

El nivel de liderazgo educativo en las IE privadas también fue evaluado y los resultados arrojaron que el $50.5 \%$ de los estudiantes, el $51.9 \%$ de los padres de familia y el $51.7 \%$ de los docentes perciben que la IE tiene un nivel de liderazgo educativo medio. En ese orden, en promedio, el $22.40 \%$ de 
Tabla 3. Valoración de la calidad educativa percibida por los estudiantes, docentes y padres de familia, por cada dimensión e institución educativa evaluada.

\begin{tabular}{|c|c|c|c|c|}
\hline Dimensión & Institución educativa & $\begin{array}{c}\text { Estudiantes } \\
(\%)\end{array}$ & $\begin{array}{l}\text { Padres de familia } \\
(\%)\end{array}$ & $\begin{array}{c}\text { Docentes } \\
(\%)\end{array}$ \\
\hline \multirow{5}{*}{$\begin{array}{l}\text { Satisfacción del } \\
\text { cliente }\end{array}$} & Santísima Virgen de las Mercedes & 30.4 & 19.1 & 28.6 \\
\hline & Blas Pascal & 21.7 & 51.1 & 14.3 \\
\hline & Eben Ezer & 21.7 & 6.4 & 21.4 \\
\hline & American School & 13 & 17 & 14.3 \\
\hline & Hans Christian Andersen & 13 & 6.4 & 21.4 \\
\hline \multirow{5}{*}{ Liderazgo educativo } & Blas Pascal & 29.1 & 49.1 & 15 \\
\hline & Eben Ezer & 27.3 & 11.3 & 30 \\
\hline & Santísima Virgen de las Mercedes & 25.5 & 17 & 20 \\
\hline & American School & 14.5 & 15.1 & 20 \\
\hline & Hans Christian Andersen & 3.6 & 7.5 & 15 \\
\hline \multirow{5}{*}{$\begin{array}{l}\text { Planeación estra- } \\
\text { tégica aplicada a la } \\
\text { educación }\end{array}$} & Santísima Virgen de las Mercedes & 37.3 & 15.8 & 20 \\
\hline & Blas Pascal & 23.5 & 50 & 26.7 \\
\hline & Eben Ezer & 17.6 & 10.5 & 20 \\
\hline & American School & 15.7 & 15.8 & 13.3 \\
\hline & Hans Christian Andersen & 5.9 & 7.9 & 20 \\
\hline \multirow{5}{*}{$\begin{array}{l}\text { Satisfacción del } \\
\text { talento humano }\end{array}$} & Blas Pascal & 33.3 & 53.8 & 20 \\
\hline & Santísima Virgen de las Mercedes & 27.8 & 23.1 & 20 \\
\hline & Eben Ezer & 19.4 & 2.6 & 20 \\
\hline & American School & 16.7 & 10.3 & 13.3 \\
\hline & Hans Christian Andersen & 2.8 & 10.3 & 26.7 \\
\hline \multirow{5}{*}{$\begin{array}{l}\text { Gestión del talento } \\
\text { humano }\end{array}$} & Santísima Virgen de las Mercedes & 35 & & 21.4 \\
\hline & Blas Pascal & 25 & & 14.3 \\
\hline & Eben Ezer & 22.5 & & 14.3 \\
\hline & American School & 17.5 & & 28.6 \\
\hline & Hans Christian Andersen & 0 & & 21.4 \\
\hline \multirow{5}{*}{ Procesos educativos } & Blas Pascal & 29.1 & 53.3 & 17.6 \\
\hline & Santísima Virgen de las Mercedes & 27.3 & 20 & 17.6 \\
\hline & Eben Ezer & 25.5 & 6.7 & 17.6 \\
\hline & American School & 12.7 & 15.6 & 23.5 \\
\hline & Hans Christian Andersen & 5.5 & 4.4 & 23.5 \\
\hline \multirow{5}{*}{$\begin{array}{l}\text { Impacto en la socie- } \\
\text { dad }\end{array}$} & Blas Pascal & 34.5 & 59.4 & 25 \\
\hline & Eben Ezer & 27.6 & 12.5 & 12.5 \\
\hline & American School & 20.7 & 9.4 & 25 \\
\hline & Santísima Virgen de las Mercedes & 17.2 & 9.4 & 25 \\
\hline & Hans Christian Andersen & 0 & 9.4 & 12.5 \\
\hline \multirow{5}{*}{$\begin{array}{l}\text { Resultados de la } \\
\text { institución educativa }\end{array}$} & Santísima Virgen de las Mercedes & 29.1 & 14 & 21.4 \\
\hline & Eben Ezer & 25.5 & 2.3 & 28.6 \\
\hline & Blas Pascal & 23.6 & 55.8 & 14.3 \\
\hline & American School & 18.2 & 18.6 & 14.3 \\
\hline & Hans Christian Andersen & 3.6 & 9.3 & 21.4 \\
\hline \multirow{5}{*}{ Recursos } & Blas Pascal & 33.3 & 42.9 & 9.00 \\
\hline & Eben Ezer & 29.6 & 14.3 & 27.3 \\
\hline & American School & 22.2 & 23.8 & 36.4 \\
\hline & Santísima Virgen de las Mercedes & 14.8 & 19 & 27.3 \\
\hline & Hans Christian Andersen & 0 & 0 & 0 \\
\hline
\end{tabular}

Fuente: Elaboración propia. 
Tabla 4. Nivel de percepción según dimensión evaluada.

\begin{tabular}{|c|c|c|c|c|}
\hline Dimensión & Tipo de cliente & Bajo (\%) & Medio (\%) & Alto (\%) \\
\hline \multirow{4}{*}{ Satisfacción del cliente } & Estudiantes & 26.7 & 50.5 & 22.8 \\
\hline & Padres de familia & 27.8 & 51.9 & 20.3 \\
\hline & Docentes & 31.0 & 48.3 & 20.7 \\
\hline & Promedio & 28.50 & 50.23 & 21.27 \\
\hline \multirow{4}{*}{ Liderazgo educativo } & Estudiantes & 26.7 & 50.5 & 22.8 \\
\hline & Padres de familia & 27.8 & 51.9 & 20.3 \\
\hline & Docentes & 24.1 & 51.7 & 24.1 \\
\hline & Promedio & 26.20 & 51.37 & 22.40 \\
\hline \multirow{4}{*}{$\begin{array}{l}\text { Planeación estratégica aplicada a la } \\
\text { educación }\end{array}$} & Estudiantes & 35.6 & 49.5 & 14.9 \\
\hline & Padres de familia & 25.3 & 50.6 & 24.1 \\
\hline & Docentes & 27.6 & 48.3 & 24.1 \\
\hline & Promedio & 29.50 & 49.47 & 21.03 \\
\hline \multirow{4}{*}{ Satisfacción del talento humano } & Estudiantes & 26.7 & 48.5 & 24.8 \\
\hline & Padres de familia & 25.3 & 58.2 & 16.5 \\
\hline & Docentes & 27.6 & 48.3 & 24.1 \\
\hline & Promedio & 26.53 & 51.67 & 21.80 \\
\hline \multirow{3}{*}{ Gestión del talento humano } & Estudiantes & 33.7 & 44.6 & 21.8 \\
\hline & Docentes & 24.1 & 51.7 & 24.1 \\
\hline & Promedio & 28.90 & 48.15 & 22.95 \\
\hline \multirow{4}{*}{ Procesos educativos } & Estudiantes & 27.7 & 48.5 & 23.8 \\
\hline & Padres de familia & 26.6 & 53.2 & 20.3 \\
\hline & Docentes & 24.1 & 51.7 & 24.1 \\
\hline & Promedio & 26.13 & 51.13 & 22.73 \\
\hline \multirow{4}{*}{ Impacto en la sociedad } & Estudiantes & 24.8 & 53.5 & 21.8 \\
\hline & Padres de familia & 25.3 & 53.2 & 21.5 \\
\hline & Docentes & 24.1 & 51.7 & 24.1 \\
\hline & Promedio & 24.73 & 52.80 & 22.47 \\
\hline \multirow{4}{*}{ Resultados de la institución educativa } & Estudiantes & 27.7 & 48.5 & 23.8 \\
\hline & Padres de familia & 29.1 & 46.8 & 24.1 \\
\hline & Docentes & 24.1 & 55.2 & 20.7 \\
\hline & Promedio & 26.97 & 50.17 & 22.87 \\
\hline \multirow{4}{*}{ Recursos } & Estudiantes & 24.8 & 52.5 & 22.8 \\
\hline & Padres de familia & 34.2 & 43.0 & 22.8 \\
\hline & Docentes & 27.6 & 48.3 & 24.1 \\
\hline & Promedio & 28.87 & 47.93 & 23.23 \\
\hline
\end{tabular}

Fuente: Elaboración propia.

los tres grupos encuestados expresó un nivel de satisfacción alto.

En cuanto al nivel de planeación estratégica aplicada a la educación en las IE, los resultados revelaron que el $49.5 \%$ de los estudiantes, el $50.6 \%$ de los padres de familia y el $48.3 \%$ de los docentes consideran que la IE tiene un nivel medio de planeación estratégica; pero, en promedio, el $21.03 \%$ de los tres grupos encuestados manifestó un nivel alto de satisfacción.

El nivel de satisfacción del talento humano en las IE privadas reveló que el $48.5 \%$ de los estudiantes, el $58.2 \%$ de los padres y el $48.3 \%$ de los docentes creen que la IE tiene un nivel de satisfacción del talento humano medio y, en promedio, el $21.80 \%$ de los tres grupos encuestados indica un alto nivel de satisfacción del talento humano. 
Para el nivel de gestión del talento humano en las IE, se obtuvo que el $44.6 \%$ de los estudiantes y el $51.7 \%$ los docentes perciben que la IE tiene un nivel medio de gestión del talento humano. En promedio, el $22.95 \%$ de los tres grupos encuestados señala un nivel alto de gestión del talento humano en las instituciones.

La encuesta sobre el nivel de procesos educativos en las IE privadas muestra que el $48.5 \%$ de los estudiantes, el $53.2 \%$ de los padres de familia y el $51.7 \%$ de los docentes encuentran que la IE tiene un nivel medio de procesos educativos. Y, en promedio, el $22.73 \%$ de los tres grupos encuestados cree que la institución tiene un alto nivel de gestión del talento humano.

Sobre el nivel de impacto que tienen las IE privadas en la sociedad, los resultados advierten que el $53.5 \%$ de los estudiantes, el $53.2 \%$ de los padres y el $51.7 \%$ de los docentes afirman que sus instituciones tienen un nivel medio de impacto en la sociedad. En promedio, el $22.47 \%$ de los tres grupos encuestados considera que poseen un alto nivel de impacto en la sociedad.

En cuanto al nivel de resultados de las IE privadas, se reveló que el $48.5 \%$ de los estudiantes, el $46.8 \%$ de los padres de familia y el $55.2 \%$ de los docentes perciben que la IE tiene un nivel medio de resultados. En promedio, el $22.87 \%$ de los encuestados confía en que la institución educativa tiene un alto nivel de resultados.

Por último, los resultados de la dimensión de recursos con los que cuentan las 5 IE arrojaron que el $52.5 \%$ de los estudiantes, el $43.0 \%$ de los padres y el $48.3 \%$ de los docentes manifiestan que la IE cuenta con un nivel medio de recursos; así, en promedio, el $23.23 \%$ de los encuestados mencionó que las IE tienen un alto nivel de recursos para brindar una elevada calidad educativa.

\section{Nivel de percepción de la calidad educativa}

En la Tabla 5, se muestra el resumen global de la calidad educativa evaluada en las $5 \mathrm{IE}$, Blas
Pascal, Santísima Virgen de las Mercedes, Eben Ezer, American School y Hans Christian Andersen, donde el $50.5 \%$ de los estudiantes, el $51.7 \%$ de los docentes y el $50.6 \%$ de los padres de familia creen que las IE antes mencionadas tienen un nivel medio de calidad educativa, mientras que, en promedio, el $24.33 \%$ percibe un alto nivel de calidad educativa.

\section{DISCUSIÓN}

Lograr la satisfacción del cliente es fundamental para cualquier organización y, en particular, la de los clientes en el ámbito educativo (alumnos y padres de familia). Con mayor razón, estos buscan satisfacer sus expectativas, porque este servicio se encuentra ligado directamente con el desarrollo personal y profesional del alumno. Es evidente que la satisfacción en el entorno educativo está relacionada con el proceso de enseñanza-aprendizaje de calidad (Osorio-Álvarez y Parra, 2016), reflejado en una buena plana docente, infraestructura adecuada, desarrollo de actividades extracurriculares y complementarias; en suma, todo aquello que conlleve formar alumnos competitivos. En esta investigación, en promedio, solo el $21.27 \%$ de los encuestados manifestó sentir una alta satisfacción, mientras que el $50.23 \%$ de los encuestados muestra una regular satisfacción. Se cree que los directivos de las IE deben trabajar fuertemente para posicionarse hacia los alumnos y padres de familia, mediante la mejora de la práctica pedagógica y acciones que conlleven a un reposicionamiento institucional.

El liderazgo es una competencia muy influyente en el éxito o el fracaso de los futuros ciudadanos. Este estudio suscribe la posición de que el liderazgo es el resultado del accionar de los directivos y docentes, quienes, a lo largo del proceso formativo, modulan en los estudiantes dicha competencia. Se observa que, en promedio, solo el $22.40 \%$ percibe que las IE desarrollan un alto nivel de liderazgo educativo. En torno a esta capacidad, los recursos y procesos influyen directamente en la calidad educativa (López, 2010). De allí que el director de cada

Tabla 5. Nivel de percepción de la calidad educativa.

\begin{tabular}{|l|l|c|c|c|}
\hline Variable & Tipo de cliente & Bajo (\%) & Medio (\%) \\
\hline \multirow{5}{*}{ Calidad educativa } & Estudiantes & 24.8 & 50.5 & 24.8 \\
\cline { 2 - 5 } & Padres de familia & 25.3 & 50.6 & 51.7 \\
\cline { 2 - 5 } & Docentes & 24.1 & 24.1 \\
\cline { 2 - 5 } & Promedio & 24.73 & 50.93 & 24.33 \\
\hline
\end{tabular}

Fuente: Elaboración propia. 
IE debe promover entre los docentes y alumnos comportamientos de equidad y justicia al momento de intervenir en la solución de los problemas. Asimismo, debe orientar y estimular el trabajo de todo el personal docente por medio de conductas motivadoras en la relación alumno-docente.

Los resultados sobre la planeación estratégica aplicada en las IE presentan, en promedio, el $21.03 \%$ de nivel alto. Este es un indicador de que en la elaboración de tales documentos de gestión no se socializa, y menos se generan compromisos entre los actores de las IE. Se sostiene que trabajar los escenarios futuros requiere, necesariamente, la participación real de los principales actores en la construcción de los diferentes proyectos (Ministerio de Educación del Perú, 2006). En ese sentido, es importante que los estudiantes, padres de familia y docentes participen en el diseño del Plan Estratégico Institucional (PEI), así como del Plan Operativo Anual (POA). El PEl, el POA y el Reglamento Interno de Trabajo (RIT) son documentos de gestión indispensables para una buena y saludable gestión institucional (Benites, 2019). De la misma forma, será importante conformar equipos de mejoramiento continuo para cada proceso.

Los directivos de cada institución educativa deben procurar mantener satisfechos a sus colaboradores. Para que los colaboradores se sientan motivados, es indispensable crear un ambiente laboral agradable y de sentido de pertenencia a la IE. Aquí, la premisa es la siguiente: a mayor satisfacción, mayor productividad y mejor desempeño del colaborador. Si bien los resultados de satisfacción y la gestión del talento humano oscilan entre el $21.80 \%$ y $22.95 \%$, respectivamente, es posible mejorar estas cifras desde la dirección de las IE, a través de acciones de valoración y reconocimiento de las capacidades de sus docentes. La dirección debe identificar las necesidades de capacitación y perfeccionamiento del personal e impulsar su desarrollo integral mediante programas de capacitación sostenibles en el tiempo, participación en proyectos, trabajo en equipo e intercambio de experiencias; se podrían, también, realizar pasantías en otras IE de referencia. Consecutivamente, se debe evaluar el desempeño del personal docente y administrativo con el reconocimiento público de quienes destaquen en tareas y responsabilidades que fortalezcan a sus instituciones.

Es claro que los procesos educativos en las IE evaluadas tienen mucho que mejorar; los resultados son evidentes, pues solo el $22.73 \%$, en promedio, manifiesta que los procesos educativos son altos y muy buenos. Al respecto, Conejeros, Rojas y Segure
(2010) dicen que, para los actores de la gestión de los procesos educativos al interior de los colegios, "la confianza no existe como pilar del sistema educativo y no se le concede el lugar que debiera tener, se le ve como una tarea aún pendiente y compleja" (p. 34). Por ello, es responsabilidad institucional y administrativa elaborar procesos claros, no engorrosos, que permitan tanto a los clientes internos como externos poder realizarlos sin ninguna dificultad. Asimismo, los procesos deben ser divulgados y visibles para los clientes. Es necesario que los estudiantes tengan conocimiento de todos los trámites administrativos que realiza la dirección y sepan sobre las mejoras de la institución. Los procesos de gestión académica y administrativa referidos deben estar alineados con el proyecto institucional y el proceso de diversificación curricular.

La responsabilidad social, traducida en el impacto que las IE tienen en la sociedad, es aún menor; en promedio, solo el $22.47 \%$ de los encuestados indicaron que es alto. Estos resultados no son alentadores para las instituciones estudiadas. Siguiendo a Héctor Mas (2014), la responsabilidad social "se consigue mediante un liderazgo que impulse la planificación y la estrategia del centro educativo, la gestión de su personal, de sus recursos y sus procesos hacia la consecución de la mejora permanente de sus resultados" (p. 36). Además, las IE se deben proyectar a la ciudadanía a través de labores de proyección y asistencia social, ayuda y voluntariado, y cuidado de las áreas verdes (parques, jardines, calles, plazas, mobiliario); asimismo, deben realizar actividades conjuntas con organismos no gubernamentales (ONG), universidades, municipalidades, iglesia u otras instituciones. Finalmente, los estudiantes deben ser motivados a participar en actividades como talleres o charlas de sensibilización, para solucionar los problemas del entorno, la delincuencia juvenil, el pandillaje, el alcoholismo, la drogadicción, los accidentes de tránsito, entre otros.

Los resultados de la institución deben ser la satisfacción plena de los docentes, alumnos y padres de familia. López (2010) reveló que la satisfacción tiene un efecto directo en los resultados de la institución y, por ende, en la calidad educativa percibida. En ese sentido, las instituciones en el estudio deben prestar especial interés a este factor, así como involucrar proyectos de investigación e innovación que les permitan mejorar su infraestructura, procesos, organización y funcionamiento; esto es, contar con buenos resultados. Para lograrlo y alcanzar los objetivos, es necesario invertir en recursos tangibles e intangibles. Es preferible que toda institución 
educativa cuente con una buena infraestructura, servicios de agua, desagüe y luz; y mantenga en perfecto estado sus laboratorios de cómputo, física y química, los cuales deben estar debidamente equipados con materiales pedagógicos, tales como pizarrones y mobiliarios. Además, deberían contar con una biblioteca completa, espacios de lectura y para las prácticas de deportes, puesto que estas son algunas de las condiciones básicas que debe cumplir una IE.

Las instituciones educativas tienen como finalidad el impulso y la orientación de la educación en sus alumnos. Una institución de calidad es aquella donde los alumnos progresan significativamente, al margen de sus posibilidades y las condiciones más apropiadas (Gento, 1996). En el mismo sentido, estimula el progreso de las capacidades como el análisis, la decisión, la investigación, el desarrollo intelectual, la autonomía humana y el espíritu crítico (Saraiva, 2008); lo que motiva al estudiante en el aprendizaje y le permite encontrarse preparado en los ámbitos científico, técnico, cultural y humano.

La calidad educativa se ve influenciada por diversos factores y variables; por ejemplo, un buen clima institucional influye significativamente en la mejora la calidad educativa (García y Pinchi, 2019). Entonces, lograr la calidad educativa es lograr formar con eficacia y eficiencia a quienes serán parte de la sociedad del presente y futuro, Por ello, se debe fomentar el desarrollo de los estudiantes en cuanto a sus competencias cognitivas, técnicas y actitudinales específicas, que les permitan insertarse asertivamente en el mercado laboral. Es fundamental que el estudiante reciba los conocimientos necesarios para saber actuar en la sociedad y convertirse en un ciudadano que aporte a su desarrollo. Es por ello que la calidad educativa tiene por objetivo satisfacer a la sociedad, asegurar el futuro y garantizar la rentabilidad de un país.

\section{CONCLUSIONES}

Después del análisis y la discusión de los resultados acerca de la calidad educativa que ofertan las IE ubicadas en el distrito de Chaclacayo, Lima, se presentan las siguientes conclusiones:

1. Los resultados de la evaluación del servicio educativo por los clientes internos (docentes) y externos (alumnos y padres de familia), basados en el modelo de calidad total que ofertan las instituciones privadas del distrito de Chaclacayo, señalan que las IE son percibidas como un servicio de alta calidad, en promedio, por el $24.3 \%$ de los encuestados. Sin embargo, existe un $50.9 \%$ del total de los encuestados que manifiesta percibir una calidad de servicio regular. Las respuestas de quienes perciben la calidad de servicio como regular, al ser analizadas en detalle, muestran comportamientos idénticos. Así, se tiene que el $51.7 \%$ de los docentes opina de este modo, al igual que el $50.5 \%$ de los estudiantes y el $50.6 \%$ de los padres de familia, en el contexto de las dimensiones: satisfacción del cliente, liderazgo educativo, planeación estratégica, satisfacción del talento humano, gestión del talento humano, procesos educativos, impacto en la sociedad, resultados de la institución educativa y recursos.

2. Lograr la calidad educativa es un reto que involucra la eficiencia y eficacia en la formación de los futuros ciudadanos del país. De acuerdo con la presente investigación, la calidad educativa es el resultado de la combinación de varios factores: liderazgo de los directivos de las IE, para crear un ambiente de trabajo horizontal y agradable; plana docente competente; infraestructura adecuada; uso de las tecnologías de información y comunicación (TIC) en el proceso de enseñanza-aprendizaje; desarrollo de actividades extracurriculares; entre otros. En este estudio, claramente se reclama a los directivos de las IE la competencia de liderazgo, principalmente por parte de los docentes y padres de familia (ver Tabla 4). El producto de la combinación de tales factores será forjar estudiantes competentes, vale decir, desarrollar sus habilidades blandas (valores de convivencia y respeto a la familia, ciudad y país) y habilidades duras (referidas a la adquisición de conocimientos para continuar sus estudios superiores tecnológicos y/o universitarios).

3. Una educación de calidad debe lograr que los alumnos reciban una formación integral (mental, social, física y espiritual), para que sean preparados para la vida y como ciudadanos de la sociedad del futuro. Esto sucede porque cada alumno deberá ser un ciudadano de bien, desarrollar emprendimientos empresariales y lograr un empleo digno, como resultado de una formación técnica o universitaria para el trabajo. Asimismo, es necesario crear en los alumnos una consciencia ecológica y de responsabilidad social, y brindarles información sobre cómo formar una familia y sostenerla en el tiempo, educando a sus hijos bajo sólidos principios; en suma, se trata de moldear ciudadanos con valores éticos. Una educación de calidad debe centrarse en una formación 
integral del futuro ciudadano, que sea líder de su comunidad y promueva cambios para asegurar la felicidad de los suyos y los demás.

4. Otro aspecto que debe destacarse está relacionado con los elementos de competitividad organizacional. Las instituciones educativas privadas ofertan servicios educativos, los cuales resultan claves para el futuro de la familia. De allí que muchos padres apuesten por que sus vástagos reciban una educación de calidad, pues entienden que la educación es la llave de éxito para su familia. Postergan muchos proyectos por financiar los estudios de sus hijos. Vista así, la oferta de las instituciones educativas está en el escrutinio permanente por los padres de familia. Si ellos perciben que su hijo no aprende, inmediatamente lo relacionarán con que la calidad del servicio es regular o mala. De la misma forma, cuando el estudiante aprende, lo relacionará con que la calidad de servicio es buena. Por ello, se enfatiza en lo importante que resulta mantener una comunicación fluida entre los padres de familia y los demás grupos de interés.

\section{REFERENCIAS BIBLIOGRÁFICAS}

[1] Barón, E. (31 de octubre de 2016). Preocupante la baja calidad educativa en América Latina: UNESCO. Educación Futura. Recuperado de http://www.educacionfutura.org/preocupantela-baja-calidad-educativa-en-america-latinaunesco.

[2] Benites, E. M. (2019). La calidad del servicio educativo en el Colegio José Quiñonez de la FAP de Lima, 2018. (Tesis de licenciatura). Universidad César Vallejo, Lima.

[3] Conejeros, M. L., Rojas, J. y Segure, T. (2010). Confianza: un valor necesario y ausente en la educación chilena. Perfiles Educativos, 32(129), 30-46. Recuperado de http://www. scielo.org.mx/pdf/peredu/v32n129/v32n129a3. pdf.

[4] García, M. E. y Pinchi, W. (2019). Clima institucional y calidad educativa en las Instituciones Educativas № 80005 y No 80077 de Educación Primaria de Trujillo. Revista Ciencia y Tecnología, 15(1), 67-74. Recuperado de http://www.revistas.unitru.edu.pe/index.php/ PGM/article/view/2295.

[5] Gento, S. (1996). Instituciones educativas para la calidad total. (Configuración de un modelo organizativo). Madrid, España: La Muralla.
[6] Gestión (3 de diciembre de 2019). Perú mejora en prueba PISA 2018, pero sigue último entre los países de la región. Gestión. Recuperado de https://gestion.pe/peru/peru-mejora-enprueba-pisa-2018-pero-sigue-ultimo-entre-lospaises-de-la-region-nndc-noticia/.

[7] Gonzales, E. (6 de abril de 2016). Problemas urgentes de la educación en el Perú. [Entrada de blog]. Descentralización. Recuperado de http://blog.pucp.edu.pe/blog/ descentralizacion/2016/04/06/problemasurgentes-de-la-educacion-en-el-peru.

[8] Hanushek, E. A. (2005). Por qué importa la calidad en la educación. Finanzas y desarrollo, 42(2), 15-19. Recuperado de https://www.imf. org/external/pubs/ft/fandd/spa/2005/06/pdf/ hanushek.pdf.

[9] Hernández-Sampieri, R., Fernández, C. y Baptista, P. (2014). Metodología de la investigación. México D. F., México: McGrawHill.

[10] La República (8 de enero de 2017). El 70\% de colegios privados de Lima no tienen adecuada infraestructura. La República. Recuperado de https://larepublica.pe/sociedad/837817el-70-de-colegios-privados-de-lima-no-tieneadecuada-infraestructura.

[11] López, P. (2010). Variables asociadas a la gestión escolar como factores de calidad educativa. Estudios Pedagógicos, 36(1), 147158. Recuperado de https://doi.org/10.4067/ S0718-07052010000100008.

[12] Mas, H. G. (2014). Modelo de evaluación basado en la calidad total para valorar la calidad educativa en la educación básica. (Tesis doctoral). Universidad Nacional de Trujillo, Trujillo. Recuperado de http:// dspace.unitru.edu.pe/bitstream/handle/ UNITRU/5618/Tesis\%20doctorado\%20-\%20 H\% c 3\%a9ctor \% 20 Grober $\% 20$ Mas $\% 20$ Sandoval.pdf?sequence=1\&isAllowed=y.

[13] Ministerio de Educación del Perú (2006). Metodología integrada para la planificación estratégica. Recuperado de http://repositorio.minedu.gob.pe/ handle/123456789/328?show=full.

[14] Ñaupas, H., Mejía, E., Novoa, E. y Villagómez, A. (2014). Metodología de la investigación cuantitativa-cualitativa y redacción de la tesis. Bogotá, Colombia: Ediciones de la U.

[15] Organisation for Economic Co-operation and Development (2019). PISA 2018: Insights and 
Interpretations. Recuperado de http://umc. minedu.gob.pe/wp-content/uploads/2019/12/ PISA-2018-Insights-and-Interpretations.pdf.

[16] Organización de las Naciones Unidas para la Educación, la Ciencia y la Cultura (2015). La educación para todos, 2000-2015: logros y desafíos. Informe de seguimiento de la EPT en el mundo, 2015. Resumen. Recuperado de http://unesdoc.unesco.org/ images/0023/002325/232565s.pdf.

[17] Organización para la Cooperación y el Desarrollo Económico (2016). Making Development Happen. Avanzando hacia una mejor educación para Perú (vol. 3). Recuperado de http://repositorio.minedu. gob.pe/bitstream/handle/MINEDU/5200/ Avanzando\%20hacia\%20una\%20mejor\%20 educaci\%c3\%b3n\%20para\%20Per\%c3\%ba. pdf? sequence $=1 \&$ isAllowed=y.

[18] Osorio-Álvarez, M. del C. y Parra, L. (2016). La satisfacción escolar en los estudiantes del primer año de la carrera de Médico Cirujano. Investigación en Educación Médica, 5(17), 3-10. Recuperado de https://doi.org/10.1016/j. riem.2015.08.002.

[19] Parodi, C. (6 de diciembre de 2013). Calidad de la Educación en el Perú. Gestión. Recuperado de https://gestion.pe/blog/ economiaparatodos/2013/12/calidad-de-laeducacion-en-el.html.
[20] Proexpansión (2014). La educación de calidad en Lima. Recuperado de https://proexpansion. com/uploads/report/pdf_file/2/Informe_ educacion_alta_.pdf.

[21] Robalino, M. (11 de julio de 2016). Perú tiene avances interesantes en el impulso de la calidad educativa. El Peruano. Recuperado de https://elperuano.pe/noticia-"peru-tieneavances-interesantes-el-impulso-de-calidadeducativa"-43625.aspx.

[22] Sánchez, G. (11 de abril de 2015). ¿Por qué no mejora la educación en América Latina? BBC. Recuperado de https://www.bbc.com/ mundo/noticias/2015/04/150409_economia_ educacion_en_america_latina_finde_bd.

[23] Saraiva, M. (2008). La calidad y los "clientes" de la enseñanza superior portuguesa. Horizontes Educacionales, 13(2), 41-54. Recuperado de https://dialnet.unirioja.es/descarga/ articulo/3987543.pdf.

[24] Vexler, I. (5 de octubre de 2016). La educación y la competitividad 2016-2017. Correo. Recuperado de https://diariocorreo. pe/opinion/la-educacion-y-la-competitivid ad-2016-2017-702501.

[25] Villarreal, E. (2014). El reto de la calidad educativa en américa latina. Recuperado de http://www.cacsla.org.mx/informacion-deinteres/el-reto-de-la-calidad-educativa-enamerica-latina. 


\title{
An Assessment of Education Quality in Private Schools
}

\author{
Rosa Katherine Cueto Huayascachi ${ }^{1}$ \\ Luis Alberto Geraldo Campos ${ }^{2}$ \\ Pedro Leonardo Tito Huamaní ${ }^{3}$
}

\begin{abstract}
The objective of this research project was to evaluate the quality of private school education in the Chaclacayo District of Lima. The study was performed with a qualitative, descriptive methodology. The quality of education at five private high schools was assessed using a sample of 209 participants, including students, teachers and parents. Three questionnaires were applied: the first with 108 questions for students, the second with 121 questions for teachers, and the third with 83 questions for parents. Results showed that the level of education at these schools was found to be "medium quality", according to the assessed criteria: customer satisfaction $(50.23 \%)$, educational leadership $(51.37 \%)$, strategic planning applied to education (48.30\%), human talent satisfaction $(51.67 \%)$, human talent management $(48.15 \%)$, teaching methods and processes $(51.13 \%)$, impact on society $(52.80 \%)$, educational outcomes $(50.17 \%)$ and educational resources $(47.93 \%)$. In conclusion, the quality of high school private education in the Chaclacayo District is found to be at a medium level, represented by a score of $50.93 \%$, according to the average perceptions of the external customers (students and parents), as well as internal customers (teachers).
\end{abstract}

Keywords: total quality; education; satisfaction; customer; leadership; management; service; talent.

\section{INTRODUCTION}

Concern for better education continues to increase, given that as societal development, historically, is based on the best and most highly-qualified members. In Latin America, the quality of education is at a standstill, as macroeconomic policy makers in most countries favor other indicators, such as the economy, production or tourism, but not quality education (Sánchez, April 11, 2015). In this context, the United Nations Educational, Scientific and Cultural Organization (UNESCO) states that education in Latin America and the Caribbean is in sharp decline, as there are at least 73 million people who barely know how to read, count and write to survive, which is a major problem for the development of the countries in this region (Barón, October 31, 2016).

The Organisation for Economic Cooperation and Development (OECD) regularly measures the state of education worldwide via the PISA test. This is the most comprehensive indicator and tool that countries and economies can use to measure education quality from an international perspective (Organisation for Economic Cooperation and Development, 2019). According to the latest PISA results, Peru is ranked 64 out of 77 countries, revealing an improvement in reading comprehension with an average score of 401, an improvement in mathematics with an average score of 400 and an improvement in science with an average score of 404 . It should be mentioned that these results place us below Argentina (Gestión, December 3, 2019). In this regard, the OECD Development Center (2016) had already stated that "Ios

1 Licenciada* in International Business and Management from the Universidad Peruana Unión (Lima, Peru). Currently working as import assistant at Import Tools. (Lima, Peru). ORCID: https://orcid.org/0000-0002-9237-9710 E-mail: rouseleo17@gmail.com

2 Licenciado* in Administration with mention in Business Management from the Universidad Peruana Unión (Lima, Peru). Currently working as research professor at the Administration Academic Program of the Universidad Peruana Unión. (Lima, Peru) ORCID: https://orcid.org/0000-0002-8366-689X E-mail: luis.geraldo@upeu.edu.pe

$3 \mathrm{PhD}$ in Administrative Sciences from the Universidad Nacional Mayor de San Marcos (Lima, Peru). Currently working as professor and research professor at UNMSM. (Lima, Peru). ORCID: https://orcid.org/0000-0002-2989-9203 E-mail: ptitoh@unmsm.edu.pe

* In Peru, Licenciatura or Titulo is a professional title granted after having defended a thesis or passing a course in accordance with the university's regulations once the undergraduate program has been completed. 
bajos resultados del país en las mediciones internacionales del aprendizaje siguen indicando que el gran reto por adelante es elevar la calidad de educación para todos los peruanos [the country's low performance in international measures of learning continues to indicate that the big challenge ahead is to improve education quality for all Peruvians]" (pp. 13-14).

Peru's situation has been improving, with an average economic growth of $5.1 \%$ between 2000 and 2015, higher than the $3.1 \%$ registered by Latin America and the Caribbean over the same period. This economic growth enabled the average citizen to achieve greater prosperity and access to opportunities. Despite this, Peru continues to face major challenges to become a country with inclusive and sustainable development (Organización para la Cooperación y el Desarrollo Económicos, 2016; Organisation for Economic Cooperation and Development, 2019). In this sense, there are also improvements in the education system, such as the population's increased access to private education, as well as the increase in the enrollment rate at the elementary level by $84 \%$ and $78 \%$ at the high school level.

Peru's investment in education has also increased, yet it is still far from significant. In terms of gross domestic product (GDP), expenditure on education rose from $2.9 \%$ in 2010 to $3.6 \%$ in 2015 ; however, the quality of education at the secondary level still remains very low (Organización para la Cooperación y el Desarrollo Económicos, 2016). In that context, the United Nations Educational, Scientific and Cultural Organization (2015) states that education is underfunded, despite budget increases in many countries. As a result, an agreement embodied in the statement "Education for All" was created in accordance with the 2030 Agenda promoted by UNESCO. According to this organization, countries should allocate $6 \%$ of GDP and/or $20 \%$ of their general budget to education. In the case of Peru, education budget represents $3.6 \%$ of GDP, a percentage far from that proposed by UNESCO (Robalino, July 11, de 2016).

According to Parodi (December 6, 2013), a quality education generates good return, both for those who receive it as well as for society itself. Indeed, "invertir en la calidad [...] reporta ventajas extraordinarias para la sociedad, [...] cómo mejorar la calidad de la educación es mucho menos evidente [investing in quality [...] has tremendous implications for society, [...] how to improve the quality of education is much less clear]" (Hanushek, 2005, p. 15).
In the same way, increasing and improving human capital enables sustainable economic growth. In this sense, countries need to work with productive and competent citizens, the outcome of a good quality education (Vexler, October 5, 2016); otherwise, they have less chance of developing their capacities. Thus, it is a question of achieving a better use of professional opportunities to successfully develop in society and environment (Villarreal, 2014). Consequently, the reality of the labor market is reflected in the fact that, due to the low level of education, of every three companies, one does not find workers with the necessary skills, which prevents access to quality employment (Gonzales, April 6, 2016).

In the Peruvian context, and more specifically in Lima, there are 5552 public schools and 11230 private schools. There are approximately one million students study in public schools and the same number in private schools (La República, January 8, 2017). Over the past twenty years, private schools, institutes and universities have grown exponentially to meet demand. However, in terms of quality accreditation and certification, according to a report by the consulting firm Proexpansión (2014), which assesses the quality of education in schools (IE, for its Spanish acronym) in Metropolitan Lima, only 200 of the 3862 schools assessed achieved quality certification. Given the need to determine the quality of education offered by these institutions, we must contextualize this for Lima in particular and for Peru in general. Thus, the assessment of private schools in the Chaclacayo District is used as a case study.

\section{METHODOLOGY}

A quantitative, descriptive approach is used in this study. This makes it possible to describe and specify the properties and important characteristics of service quality in private sector educational institutions in the Chaclacayo District, in 2018. It is also a non-experimental study because variables were not deliberately manipulated, as phenomena were only observed in their natural context and subsequently analyzed. Likewise, it is a cross-sectional study, because information was collected at a specific time (Hernández-Sampieri, Fernández \& Baptista, 2014).

\section{Sampling}

Initially, the population was made up of 15 private schools located in the Chaclacayo District. Non-probability sampling technique was used, in which the judgments of the research team prevails, as proposed by Ñaupas, Mejía, Novoa \& Villagómez (2014). It should be noted that the private schools 
in this study were the only schools that agreed to apply the instruments. It should be also noted that 15 private schools in the Chaclacayo District were visited and permission to apply the three questionnaires of the study was agreed to by their leadership. However, during fieldwork, only 5 schools applied the questionnaires. This fact does not diminish the reliability of the study, the breakdown of which is shown in Table 1.

Table 1. Sample distribution.

\begin{tabular}{|l|c|}
\hline \multicolumn{1}{|c|}{ Group } & Sample \\
\hline Students in the 4th and 5th year of high school ${ }^{\mathrm{TN}}$ & 101 \\
\hline Parents & 79 \\
\hline Teachers & 29 \\
\hline Total & 209 \\
\hline
\end{tabular}

Source: Prepared by the authors.

$\mathrm{TN}$ : In Peru, secondary education is comprised of five academic years.

\section{Measuring instruments and Data analysis}

For this study, three questionnaires developed by the psychopedagogue Héctor Mas (2014) and designed for students, teachers and parents were considered.

The first instrument, directed at students, consists of 9 dimensions (customer satisfaction, leadership, strategic planning, human talent satisfaction, human talent management, teaching methods and processes, impact on society, educational outcomes and educational resources), with 108 items and a Cronbach alpha reliability of 0.969 . It has multiple choice questions and uses the Likert scale, where 4 is equal to always and 1 is equal to never (Mas, 2014). A Cronbach's alpha reliability of 0.973 was obtained (see Table 2).

The second instrument, directed at teachers, consists of 9 dimensions, with 121 items and a Cronbach's alpha reliability of 0.962 . It has multiple choice questions and uses the Likert scale, where 4 equals always and 1 equals never. A Cronbach's alpha reliability of 0.989 was obtained (see Table 2 ).

The third instrument, directed at parents, consists of 8 dimensions (customer satisfaction, leadership, strategic planning, human talent satisfaction, teaching methods and processes, impact on society, educational outcomes and educational resources), with 83 items and a Cronbach's alpha reliability of 0.956 . A Cronbach's alpha reliability of 0.979 was obtained (see Table 2).
Table 2. Reliability statistics.

\begin{tabular}{|c|c|c|}
\hline Instruments & Cronbach's alpha & No. of items \\
\hline Students & 0.973 & 108 \\
\hline Teachers & 0.989 & 121 \\
\hline Parents & 0.979 & 83 \\
\hline
\end{tabular}

Source: Prepared by the authors.

Subsequently, descriptive and frequency statistics of each item were analyzed, with which results were obtained according to the instrument applied. Then, the calculations of variables were conducted to weigh each dimension, in order to conduct the cross-tabulation analysis and, thus, to obtain the levels of each dimension per instrument. Finally, the dimensions and their respective weight were calculated to determine level of educational quality, according to the perceptions of students, teachers and parents.

\section{RESULTS}

Assessment of education quality as perceived by students, teachers and parents per dimension and school assessed

In this section, a comparative table containing all the schools assessed is presented in order to identify which one has a higher level of satisfaction in educational quality as perceived by students, teachers and parents, is shown.

Table 3 shows the satisfaction level of students, parents and teachers for each dimension per school. The values obtained for "customer satisfaction" dimension were the following. At IE Santísima Virgen de las Mercedes, based on a sample of 60 respondents, $30.4 \%$ of students, $28.6 \%$ of teachers and $19.1 \%$ of parents were satisfied. At IE Blas Pascal, based on a sample of 55 respondents, $51.1 \%$ of parents, $21.7 \%$ of students and $14.3 \%$ of teachers, were satisfied. At IE Eben Ezer, based on a sample of 51 respondents, $21.7 \%$ of students, $21.4 \%$ teachers and $64 \%$ of parents were satisfied. At IE American School, based on a sample of 29 respondents, $17 \%$ of parents, $14.3 \%$ teachers and $13 \%$ of students were satisfied. Finally, at IE Hans Christian Andersen, based on a sample of 14 respondents, $21.4 \%$ of teachers, $13 \%$ students and only $6.4 \%$ of parents were satisfied.

Perception regarding "education leadership" dimension, assessed in the five schools mentioned, varies according to the studied groups. At IE Blas Pascal, $49.1 \%$ of parents, $29.1 \%$ of students and only $15 \%$ of teachers, perceive education leadership within 
the school. At IE Eben Ezer, 30\% of teachers, 27\% of students and only $11.3 \%$ of parents perceive education leadership within the school. At IE Santísima Virgen de las Mercedes, $25.5 \%$ of students, $20 \%$ of teachers and $17 \%$ of parents perceive education leadership within the school. At IE American School, $20 \%$ of teachers, $15.1 \%$ of parents and $14.5 \%$ of students perceive education leadership within the school. Finally, at IE Hans Christian Andersen, 15\% of teacher, $7.5 \%$ of parents and $3.6 \%$ of students perceive education leadership within the school.

Perception regarding "strategic planning applied to education" dimension was also rated by the three groups in the five schools mentioned. At IE Santísima Virgen de las Mercedes, $37.3 \%$ of students, $20 \%$ of teachers and $15.8 \%$ of parents consider that there is strategic planning in the school. At IE Blas Pascal, $50 \%$ of parents, $26.7 \%$ of teachers and $17.6 \%$ of students consider that there is strategic planning in the school. At IE Eben Ezer, 20\% of teachers, $17.6 \%$ students and $10.5 \%$ of parents consider that there is strategic planning in the school. At IE American School, 15.8\% of parents, $15.7 \%$ of students and $13.3 \%$ of teachers consider that there is educational planning in the school. Finally, at IE Hans Christian Andersen, $20 \%$ of teachers, $7.9 \%$ of parents and $5.9 \%$ of students consider that there is strategic planning applied to education in the school.

Perception regarding "human talent satisfaction" dimension, assessed in the five schools mentioned, varies according to the studied groups. At IE Blas Pascal, $53.8 \%$ of parents, $33.3 \%$ of students and $20 \%$ of teachers perceive satisfaction among school staff members. At IE Santísima Virgen de las Mercedes, $27.8 \%$ of students, $23.1 \%$ of parents and $20 \%$ of teachers perceive satisfaction among school staff members. At IE Eben Ezer, $20 \%$ of teachers, $19.4 \%$ of students and only $2.6 \%$ of parents perceive satisfaction among school staff members. At IE American School, $16.7 \%$ of students, $13.3 \%$ of teachers and only $10.3 \%$ of parents perceive satisfaction among school staff members. Finally, at IE Hans Christian Andersen, $26.7 \%$ of teachers, only $10.3 \%$ of parents and $2.8 \%$ of students perceive satisfaction among school staff members.

Questions regarding "human talent management" dimension were only addressed to students and teachers, as these two groups spend more time in the school. At IE Santísima Virgen de las Mercedes, $35 \%$ of students and $21.4 \%$ of teachers perceive human talent management. Results were lower at IE Blas Pascal, where $25 \%$ of students and only $14.3 \%$ of teachers perceive human talent management. At
IE American School, $28.6 \%$ of teachers and $17.5 \%$ of students perceive human talent management, whereas, at IE Hans Christian Andersen, only 21.4\% of teachers perceive human talent management.

"Teaching methods and processes" are essential elements within schools; therefore, it was decided to measure this dimension in the assessment conducted on the five schools. In that regard, at IE Blas Pascal, $53.3 \%$ of parents, $29.1 \%$ of students and $17.6 \%$ of teachers consider that there are teaching methods and processes in place. At IE Santísima Virgen de las Mercedes, $27.3 \%$ of students, $20 \%$ of parents and $17.6 \%$ of teachers consider that there are teaching methods and processes in place. At IE Eben Ezer, $25.5 \%$ of students, $17.6 \%$ of teachers and only $6.7 \%$ of parents consider there are teaching methods and processes in place. At IE American School, $25 \%$ of teachers, $15.6 \%$ of parents and $12.7 \%$ of students consider that there are teaching methods and processes in place. Finally, at IE Hans Christian Andersen, $23.5 \%$ of teachers consider that there are teaching methods and processes in place, whereas only $5.5 \%$ of students and $4.4 \%$ of parents share that opinion.

"Impact on society" is another dimension of great importance for the assessment of schools, for this reason, it was evaluated from the perspective of students, parents and teachers. In that sense, at IE Blas Pascal, $59.5 \%$ of parents, $34.5 \%$ of students and $25 \%$ of teachers believe the school has an impact on society. At IE Eben Ezer, $27.6 \%$ of students and $12.5 \%$ of parents and teachers believe the school has an impact on society. At IE American School, $25 \%$ of teachers, $20.7 \%$ of students and $9.4 \%$ of parents believe the school has an impact on society. At IE Santísima Virgen de las Mercedes, $25 \%$ of teachers, $17.2 \%$ of students and only $9.4 \%$ of parents believe the school has an impact on society. Finally, at IE Hans Christian Andersen, 12.5\% of teachers and $9.4 \%$ of parents believe the school has an impact on society.

The quality of education is also reflected on the educational outcomes of each school; therefore, it is important to know the outcomes obtained by the schools assessed and whether they are known by the respondents. In that regard, at IE Santísima Virgen de las Mercedes, 29\% of students, $21.4 \%$ of teachers and only $2.3 \%$ of parents are aware of the school educational outcomes. At IE Eben Ezer, $28.6 \%$ of teachers, $25.5 \%$ of students and only $2.3 \%$ of parents are aware of the school educational outcomes. In contrast, at IE Blas Pascal, numbers are encouraging as $55.8 \%$ of parents, $23.6 \%$ of students and $14.3 \%$ of teachers express they 
are aware of the school educational outcomes. At IE American School, $18.6 \%$ of parents, $18.2 \%$ of students and $14.3 \%$ of teachers are aware of the school educational outcomes. Finally, at IE Hans Christian Andersen, 21.4\% of teachers, 9.3\% of parents and only $3.6 \%$ of students, respectively, are aware of the school educational outcomes.

Finally, "educational resources" dimension was considered in order to determine whether the school has enough resources to provide a good service. At IE Blas Pascal, $42.9 \%$ of parents, $33.3 \%$ of students and only $9 \%$ of teachers mention that the school has enough resources to provide a good education. At IE Eben Ezer, $29.6 \%$ of students, $27.3 \%$ of teachers and $14.3 \%$ of parents mention that the school has enough resources to provide a good education. At IE American School, $36.4 \%$ of teachers, $23.8 \%$ of parents and $22.2 \%$ of students mention that the school has enough resources to provide a good education. At IE Santísima Virgen de las Mercedes, $27.3 \%$ of teachers, $19 \%$ of parents and $14.8 \%$ of students mention that the school has enough resources to provide a good education. It is worth mentioning the overall results for IE Hans Christian Andersen regarding this dimension are minimal, consequently, the school must continue working to increase its resources and provide a good service.

Therefore, IE Blas Pascal and IE Santísima Virgen de las Mercedes are the schools that top the list of good quality education providers. That is to say, these two schools are the ones that provide the highest educational quality according to students, teachers and parents; therefore, they are the ones that offer the greatest satisfaction (see Table 3).

\section{Perception level per each assessed dimension}

Table 4 shows the perception level of students, teachers and parents in regards to education quality. The overall analysis of the 5 private schools found that $50.5 \%$ of students, $51.9 \%$ of parents and $48.3 \%$ of teachers have a medium level of satisfaction regarding education quality; whereas, on average, $21.27 \%$ of respondents show a high level of satisfaction regarding education quality.

The assessment of education leadership in private schools found that $50.5 \%$ of students, $51.9 \%$ parents and $51.7 \%$ of teachers perceive that the school has a medium level of education leadership; whereas, on average, $22.40 \%$ of respondents show a high level of satisfaction regarding education leadership.

The assessment of strategic planning applied to education in schools found that $49.5 \%$ of students,
$50.6 \%$ of parents and $48.3 \%$ of teachers believe that the school has a medium level of strategic planning; whereas, on average, $21.03 \%$ of respondents show a high level of satisfaction regarding strategic planning.

The assessment of human talent satisfaction in private schools found that $48.5 \%$ of students, $58.2 \%$ of parents and $48.3 \%$ of teachers believe that the school has a medium level of satisfaction regarding human talent satisfaction; whereas, on average, $21.80 \%$ of respondents show a high level of satisfaction regarding human talent.

The assessment of human talent management in private schools found that $44.6 \%$ of students and $51.7 \%$ of teachers perceive a medium level of human talent management; whereas, on average, $22.95 \%$ of respondents perceive a high level of human talent management in their schools.

The assessment of teaching methods and processes in private schools found that $48.5 \%$ of students, $53.2 \%$ of parents and $51.7 \%$ of teachers, respectively, consider that the school has a medium level of teaching methods and processes; whereas, on average, $22.73 \%$ of respondents consider that the schools have a high level of teaching methods and processes.

The assessment of the impact of private schools on society found that $53.5 \%$ of students, $53.2 \%$ of parents and $51.7 \%$ of teachers consider that their schools have a medium impact on society; whereas, on average, $22.47 \%$ of respondents consider the schools have a high impact on society.

The assessment of private schools' educational outcomes found that $48.5 \%$ of students, $46.8 \%$ of parent and $55.2 \%$ of teachers consider that the school has a medium level regarding results; whereas, on average, $22.87 \%$ of respondents believes that the school has a high level of results.

Lastly, the assessment of resources conducted on five private schools found that $52.5 \%$ of students, $43.0 \%$ of parents and $48.3 \%$ of teachers state that the school has a medium level of educational resources; whereas, on average, $23.23 \%$ of respondents stated that the schools have a high level of educational resources enough to provide a good quality education.

\section{Level of perception of education quality}

Table 5 shows the overall summary of education quality assessed in the five schools: IE Blas Pascal, IE Santísima Virgen de las Mercedes, IE Eben 
Table 3. Assessment of education quality as perceived by students, teachers and parents per dimension and school assessed.

\begin{tabular}{|c|c|c|c|c|}
\hline Dimension & School & Students (\%) & Parents (\%) & Teachers (\%) \\
\hline \multirow{5}{*}{ Customer satisfaction } & IE Santísima Virgen de las Mercedes & 30.4 & 19.1 & 28.6 \\
\hline & IE Blas Pascal & 21.7 & 51.1 & 14.3 \\
\hline & IE Eben Ezer & 21.7 & 6.4 & 21.4 \\
\hline & IE American School & 13 & 17 & 14.3 \\
\hline & IE Hans Christian Andersen & 13 & 6.4 & 21.4 \\
\hline \multirow{5}{*}{ Education leadership } & IE Blas Pascal & 29.1 & 49.1 & 15 \\
\hline & IE Eben Ezer & 27.3 & 11.3 & 30 \\
\hline & IE Santísima Virgen de las Mercedes & 25.5 & 17 & 20 \\
\hline & IE American School & 14.5 & 15.1 & 20 \\
\hline & IE Hans Christian Andersen & 3.6 & 7.5 & 15 \\
\hline \multirow{5}{*}{$\begin{array}{l}\text { Strategic planning } \\
\text { applied to education }\end{array}$} & IE Santísima Virgen de las Mercedes & 37.3 & 15.8 & 20 \\
\hline & IE Blas Pascal & 23.5 & 50 & 26.7 \\
\hline & IE Eben Ezer & 17.6 & 10.5 & 20 \\
\hline & IE American School & 15.7 & 15.8 & 13.3 \\
\hline & IE Hans Christian Andersen & 5.9 & 7.9 & 20 \\
\hline \multirow{5}{*}{$\begin{array}{l}\text { Human talent satis- } \\
\text { faction }\end{array}$} & IE Blas Pascal & 33.3 & 53.8 & 20 \\
\hline & IE Santísima Virgen de las Mercedes & 27.8 & 23.1 & 20 \\
\hline & IE Eben Ezer & 19.4 & 2.6 & 20 \\
\hline & IE American School & 16.7 & 10.3 & 13.3 \\
\hline & IE Hans Christian Andersen & 2.8 & 10.3 & 26.7 \\
\hline \multirow{5}{*}{$\begin{array}{l}\text { Human talent mana- } \\
\text { gement }\end{array}$} & IE Santísima Virgen de las Mercedes & 35 & & 21.4 \\
\hline & IE Blas Pascal & 25 & & 14.3 \\
\hline & IE Eben Ezer & 22.5 & & 14.3 \\
\hline & IE American School & 17.5 & & 28.6 \\
\hline & IE Hans Christian Andersen & 0 & & 21.4 \\
\hline \multirow{5}{*}{$\begin{array}{l}\text { Teaching methods } \\
\text { and processes }\end{array}$} & IE Blas Pascal & 29.1 & 53.3 & 17.6 \\
\hline & IE Santísima Virgen de las Mercedes & 27.3 & 20 & 17.6 \\
\hline & IE Eben Ezer & 25.5 & 6.7 & 17.6 \\
\hline & IE American School & 12.7 & 15.6 & 23.5 \\
\hline & IE Hans Christian Andersen & 5.5 & 4.4 & 23.5 \\
\hline \multirow{5}{*}{ Impact on society } & IE Blas Pascal & 34.5 & 59.4 & 25 \\
\hline & IE Eben Ezer & 27.6 & 12.5 & 12.5 \\
\hline & IE American School & 20.7 & 9.4 & 25 \\
\hline & IE Santísima Virgen de las Mercedes & 17.2 & 9.4 & 25 \\
\hline & IE Hans Christian Andersen & 0 & 9.4 & 12.5 \\
\hline \multirow{5}{*}{ Educational outcomes } & IE Santísima Virgen de las Mercedes & 29.1 & 14 & 21.4 \\
\hline & IE Eben Ezer & 25.5 & 2.3 & 28.6 \\
\hline & IE Blas Pascal & 23.6 & 55.8 & 14.3 \\
\hline & IE American School & 18.2 & 18.6 & 14.3 \\
\hline & IE Hans Christian Andersen & 3.6 & 9.3 & 21.4 \\
\hline \multirow{5}{*}{$\begin{array}{l}\text { Educational resour- } \\
\text { ces }\end{array}$} & IE Blas Pascal & 33.3 & 42.9 & 9.00 \\
\hline & IE Eben Ezer & 29.6 & 14.3 & 27.3 \\
\hline & IE American School & 22.2 & 23.8 & 36.4 \\
\hline & IE Santísima Virgen de las Mercedes & 14.8 & 19 & 27.3 \\
\hline & IE Hans Christian Andersen & 0 & 0 & 0 \\
\hline
\end{tabular}

Source: Prepared by the authors. 
Table 4. Level of perception per assessed dimension.

\begin{tabular}{|c|c|c|c|c|}
\hline Dimension & Type of customer & Low (\%) & Medium(\%) & High (\%) \\
\hline \multirow{4}{*}{ Customer satisfaction } & Students & 26.7 & 50.5 & 22.8 \\
\hline & Parents & 27.8 & 51.9 & 20.3 \\
\hline & Teachers & 31.0 & 48.3 & 20.7 \\
\hline & Average & 28.50 & 50.23 & 21.27 \\
\hline \multirow{4}{*}{ Education leadership } & Students & 26.7 & 50.5 & 22.8 \\
\hline & Parents & 27.8 & 51.9 & 20.3 \\
\hline & Teachers & 24.1 & 51.7 & 24.1 \\
\hline & Average & 26.20 & 51.37 & 22.40 \\
\hline \multirow{4}{*}{ Strategic planning applied to education } & Students & 35.6 & 49.5 & 14.9 \\
\hline & Parents & 25.3 & 50.6 & 24.1 \\
\hline & Teachers & 27.6 & 48.3 & 24.1 \\
\hline & Average & 29.50 & 49.47 & 21.03 \\
\hline \multirow{4}{*}{ Human talent satisfaction } & Students & 26.7 & 48.5 & 24.8 \\
\hline & Parents & 25.3 & 58.2 & 16.5 \\
\hline & Teachers & 27.6 & 48.3 & 24.1 \\
\hline & Average & 26.53 & 51.67 & 21.80 \\
\hline \multirow{3}{*}{ Human talent management } & Students & 33.7 & 44.6 & 21.8 \\
\hline & Teachers & 24.1 & 51.7 & 24.1 \\
\hline & Average & 28.90 & 48.15 & 22.95 \\
\hline \multirow{4}{*}{ Teaching methods and processes } & Students & 27.7 & 48.5 & 23.8 \\
\hline & Parents & 26.6 & 53.2 & 20.3 \\
\hline & Teachers & 24.1 & 51.7 & 24.1 \\
\hline & Average & 26.13 & 51.13 & 22.73 \\
\hline \multirow{4}{*}{ Impact on society } & Students & 24.8 & 53.5 & 21.8 \\
\hline & Parents & 25.3 & 53.2 & 21.5 \\
\hline & Teachers & 24.1 & 51.7 & 24.1 \\
\hline & Average & 24.73 & 52.80 & 22.47 \\
\hline \multirow{4}{*}{ Educational outcomes } & Students & 27.7 & 48.5 & 23.8 \\
\hline & Parents & 29.1 & 46.8 & 24.1 \\
\hline & Teachers & 24.1 & 55.2 & 20.7 \\
\hline & Average & 26.97 & 50.17 & 22.87 \\
\hline \multirow{4}{*}{ Educational resources } & Students & 24.8 & 52.5 & 22.8 \\
\hline & Parents & 34.2 & 43.0 & 22.8 \\
\hline & Teachers & 27.6 & 48.3 & 24.1 \\
\hline & Average & 28.87 & 47.93 & 23.23 \\
\hline
\end{tabular}

Source: Prepared by the authors.

Ezer, IE American School and IE Hans Christian Andersen. Results show that $50.5 \%$ of students, $51.7 \%$ of teachers and $50.6 \%$ of parents believe that the above-mentioned schools offer a medium quality education; whereas, on average, $24.33 \%$ of respondents consider that said schools offer a high quality education.

\section{DISCUSSION}

Achieving customer satisfaction is fundamental for any organization and, in particular, that of customers in the educational field (students and parents). Schools seek to satisfy customer expectations, as education is directly linked to a student's personal 
and professional development. It is clear that satisfaction in the educational environment is related to the quality teaching-learning process (Osorio-Álvarez \& Parra, 2016), reflected in good teaching staff, adequate infrastructure, and extracurricular and supplementary activities; in short, everything that involves shaping competitive students. In this investigation, $21.27 \%$ of respondents expressed high satisfaction, while $50.23 \%$ of respondents expressed a medium level of satisfaction. It is believed that school authorities should work diligently to reach students and parents by improving pedagogical practices and actions that result in a positive repositioning of the school.

Leadership is a very significant competency in the success or failure of future citizens. This study affirms that leadership is the result of the actions of school authorities and teachers, who, throughout the training process, instill this competence in students. It is observed that, on average, only $22.40 \%$ of respondents perceive that schools have a high level of education leadership. In addition to leadership, resources and processes directly influence educational quality (López, 2010). Therefore, principals should promote equity and justice among teachers and students when solving problems. Likewise, they should guide and encourage the work of teaching staff by setting the example in regards to the student-teacher relationship.

On average, only $21.03 \%$ of respondents consider that schools have a high level of strategic planning applied to education. This is an indicator that when the contents of strategic planning documents are not shared, even less collaboration is generated among school stakeholders. It is argued that planning future scenarios necessarily requires the effective participation of the main parties in the development of education projects (Ministerio de Educación del Perú, 2006). Therefore, it is important that students, parents and teachers participate in the designing of the Institutional Strategic Plan (PEI, for its Spanish acronym), as well as the Annual Operational Plan (POA, for its Spanish acronym). The PEI, the POA and the Internal Labor Regulations (RIT, for its Spanish acronym) are essential documents for good and healthy institutional management (Benites, 2019). Similarly, it is important to create continuous improvement teams for each process.

School principals should attempt to keep their employees satisfied. For employees to feel motivated, it is essential to create a pleasant working environment and a sense of belonging to the school. The premise is: the greater the satisfaction, the greater the productivity and the better the performance of employees. Although the results regarding satisfaction and management of human talent vary between $21.80 \%$ and $22.95 \%$, respectively, it is possible to improve these figures from the school management, through actions that express appreciation and recognition of the capacities of teachers. Management should identify the training and development needs of staff and promote their comprehensive development through time-sustainable training programs, project participation, teamwork and exchange of experiences. Internships in different schools may also be a training option. Accordingly, teaching and administrative staff performance should be evaluated and those who excel in tasks and responsibilities that strengthen their schools should be publicly acknowledged.

It is clear that the teaching methods and processes in the schools assessed have much to improve. Results reflect that only $22.73 \%$ of respondents consider teaching methods and processes to be of high level and very good. In this regard, Conejeros, Rojas and Segure (2010) state that, for parties involved in the management of teaching methods and processes within schools, "la confianza no existe como pilar del sistema educativo y no se le concede el lugar que debiera tener, se le ve como una tarea aún pendiente y compleja [trust does not exist as a pillar of the education system and is not given the place it should have, it is seen as a still pending and complex task]" ( $p$. 34). Therefore, it is an institutional and administrative responsibility to develop clear, not burdensome processes that allow both internal and external customers to conduct them without any difficulty. The mentioned academic and administrative management processes

Table 5. Level of perception of education quality

\begin{tabular}{|c|c|c|c|c|}
\hline Variable & Type of customer & Low (\%) & Medium (\%) & High (\%) \\
\hline \multirow{4}{*}{ Education quality } & Students & 24.8 & 50.5 & 24.8 \\
\cline { 2 - 5 } & Parents & 25.3 & 50.6 & 24.1 \\
\cline { 2 - 5 } & Teachers & 24.1 & 51.7 & 24.1 \\
\cline { 2 - 5 } & Average & 24.73 & 50.93 & 24.33 \\
\hline
\end{tabular}

Source: Prepared by the authors. 
must be aligned with the institutional project and the process of curricular diversification.

The perception level of social responsibility, translated into the impact a school has on society, is even lower. On average, only $22.47 \%$ of respondents indicated that it is high. These results are not encouraging for the institutions studied. According to Héctor Mas (2014), social responsibility "se consigue mediante un liderazgo que impulse la planificación y la estrategia del centro educativo, la gestión de su personal, de sus recursos y sus procesos hacia la consecución de la mejora permanente de sus resultados [is achieved through a leadership that drives planning and strategy, personnel management, resources and processes of educational centers towards the achievement of the permanent improvement of results]" (p. 36). In addition, schools should be engaged with the public through awareness and social work, volunteering, and care of green spaces (parks, gardens, streets, squares) and outdoor furniture. They should also engage in joint activities with non-governmental agencies (NGOs), universities, municipalities, churches or other institutions. Finally, students should be motivated to participate in activities, such as workshops or awareness talks, to solve problems related to their surroundings, juvenile delinquency, gangs, alcoholism, drug addiction, traffic accidents, and other problems.

Results achieved by schools should be the full satisfaction of teachers, students and parents. López (2010) revealed that satisfaction has a direct effect on schools' results and, therefore, on perceived educational quality. In this sense, the schools in this study should place a particular focus on this factor; they should also adopt research and innovation projects which enable them to improve their infrastructure, processes, organization and operation, that is, to have good results. To do so and achieve the set goals, it is necessary to invest in tangible and intangible resources. Every school should have good infrastructure, water, drainage and electricity services, and maintain in perfect order its computer, physical and chemical laboratories which should be properly equipped with teaching materials, such as blackboards and desks. In addition, they should have a complete library, spaces for reading and for sports, as these are some of the basic requirements that schools must meet.

Schools have the purpose to promote and orient the education of their pupils. A quality educational institution is one where students progress significantly, regardless of financial situation and surroundings (Gento, 1996). In that sense, education quality stimulates the improvement of capacities such as analysis, decision-making, research, intellectual development, human autonomy and critical thinking (Saraiva, 2008), which promotes learning in students and prepares them for scientific, technical, cultural and human fields.

Educational quality is influenced by various factors and variables; for example, a good institutional climate significantly influences the improvement of educational quality (García \& Pinchi, 2019). Thus, achieving educational quality is to effectively and efficiently educate those who will be part of the present and future society. Therefore, students' development in terms of their specific cognitive, technical and attitudinal skills, which allow them to insert assertively into the labor market, should be encouraged. It is essential that students receive the necessary knowledge to know how to behave in society and become citizens who contribute to its development. It is for this reason that the objective of education quality is to satisfy society, ensure the future and ensure the financial success of a country.

\section{CONCLUSIONS}

After the analysis and discussion of the results on the quality of education offered by the schools in the Chaclacayo District of Lima, the following conclusions are presented:

1. The assessment on education quality as perceived by internal (teachers) and external customers (students and parents), based on the total quality model offered by private schools in the Chaclacayo District, show that $24.3 \%$ respondents believe that schools provide a high quality education. However, $50.9 \%$ of respondents say they perceive a medium level of education quality. The responses of those who have this perception, when analyzed in detail, show identical behaviors. Thus, $51.7 \%$ of teachers, $50.5 \%$ of students and $50.6 \%$ of parents share this opinion regarding all dimensions: customer satisfaction, educational leadership, strategic planning, human talent satisfaction, human talent management, teaching methods and processes, impact on society, educational outcomes and educational resources.

2. Achieving quality in education is a challenge that involves efficiency and effectiveness in the training of the country's future citizens. According to this research, quality education results from the combination of several factors: school leadership, to create a same-level and pleasant working environment; competent teaching staff; adequate 
infrastructure; use of information and communication technologies (ICT) in the teaching-learning process; development of extracurricular activities; among others. In this study, school managers are being asked, mainly by teachers and parents to be leaders (see Table 4). The combination of such factors will result in competent students, that is, students who will develop their soft skills (values of coexistence and respect for family, city and country) and hard skills (referring to the acquisition of knowledge to continue their higher and/or university education).

3. Quality education must ensure that students receive a comprehensive education (mental, social, physical and spiritual), so that they are prepared for life and as future citizens of society. This is because each student must be a good citizen, develop entrepreneurial ventures and achieve a respectable profession, as a result of technical or university training. It should also instill ecological and social responsibility awareness among students and provide them with information on how to form and support a family over time, educating their children according to sound principles. In short, it is about shaping citizens with ethical values. A quality education must focus on the comprehensive education of future citizens, who will be the leaders of their community and promote changes to ensure their own happiness and that of others.

4. Another aspect to be highlighted is related to the elements of organizational competitiveness. Private schools offer educational services, which are key to the future of the family. Many parents decide that their children receive a good quality education, because they understand that education is the key to the success of their family. They put off many projects to fund their children's studies. Thus, the educational offer of schools is under permanent scrutiny by parents. If they perceive that their child does not learn, they will immediately relate it to the fact that education quality is medium or poor. Similarly, when the child learns, they will relate it to a good quality education. Therefore, the importance of maintaining constant communication between parents and other interest groups is emphasized.

\section{REFERENCES}

[1] Barón, E. (October 31, 2016). Preocupante la baja calidad educativa en América Latina: UNESCO. Educación Futura. Retrieved from http://www.educacionfutura.org/preocupantela-baja-calidad-educativa-en-america-latinaunesco.

[2] Benites, E. M. (2019). La calidad del servicio educativo en el Colegio José Quiñonez de la FAP de Lima, 2018. (Degree thesis). Universidad César Vallejo, Lima.

[3] Conejeros, M. L., Rojas, J. \& Segure, T. (2010). Confianza: un valor necesario y ausente en la educación chilena. Perfiles Educativos, 32(129), 30-46. Retrieved from .

[4] García, M. E. \& Pinchi, W. (2019). Clima institucional y calidad educativa en las Instituciones Educativas No 80005 y No 80077 de Educación Primaria de Trujillo. Revista Ciencia y Tecnología, 15(1), 67-74. Retrieved from http://www.revistas.unitru.edu.pe/index. php/PGM/article/view/2295.

[5] Gento, S. (1996). Instituciones educativas para la calidad total. (Configuración de un modelo organizativo). Madrid, Spain: La Muralla.

[6] Gestión (December 3, 2019). Perú mejora en prueba PISA 2018, pero sigue último entre los países de la región. Gestión. Retrieved from https://gestion.pe/peru/peru-mejora-enprueba-pisa-2018-pero-sigue-ultimo-entre-lospaises-de-la-region-nndc-noticia/.

[7] Gonzales, E. (April 6, 2016). Problemas urgentes de la educación en el Perú. [Blog entry]. Descentralización. Retrieved from http://blog.pucp.edu.pe/blog/ descentralizacion/2016/04/06/problemasurgentes-de-la-educacion-en-el-peru.

[8] Hanushek, E. A. (2005). Por qué importa la calidad en la educación. Finanzas y desarrollo, 42(2), 15-19. Retrieved from https://www.imf. org/external/pubs/ft/fandd/spa/2005/06/pdf/ hanushek.pdf.

[9] Hernández-Sampieri, R., Fernández, C. \& Baptista, P. (2014). Metodología de la investigación. México D. F., Mexico: McGrawHill.

[10] La República (January 8, 2017). El 70\% de colegios privados de Lima no tienen adecuada infraestructura. La República. Retrieved from https://larepublica.pe/sociedad/837817-el70-de-colegios-privados-de-lima-no-tieneadecuada-infraestructura.

[11] López, P. (2010). Variables asociadas a la gestión escolar como factores de calidad educativa. Estudios Pedagógicos, 36(1), 147- 
158. Retrieved from https://doi.org/10.4067/ S0718-07052010000100008.

[12] Mas, H. G. (2014). Modelo de evaluación basado en la calidad total para valorar la calidad educativa en la educación básica. (Doctoral thesis). Universidad Nacional de Trujillo, Trujillo. Retrieved from http:// dspace.unitru.edu.pe/bitstream/handle/ UNITRU/5618/Tesis\%20doctorado\%20-\%20 $\mathrm{H} \%$ c 3\%a9ctor $\% 20$ Grober $\% 20$ Mas $\% 20$ Sandoval. pdf? sequence $=1$ \&isAllowed $=y$.

[13] Ministerio de Educación del Perú (2006). Metodología integrada para la planificación estratégica. Retrieved from http://repositorio.minedu.gob.pe/ handle $/ 123456789 / 328$ ?show=full.

[14] Ñaupas, H., Mejía, E., Novoa, E. \& Villagómez, A. (2014). Metodología de la investigación cuantitativa-cualitativa y redacción de la tesis. Bogotá, Colombia: Ediciones de la U.

[15] Organisation for Economic Co-operation and Development (2019). PISA 2018: Insights and Interpretations. Retrieved from http://umc. minedu.gob.pe/wp-content/uploads/2019/12/ PISA-2018-Insights-and-Interpretations.pdf.

[16] Organización de las Naciones Unidas para la Educación, la Ciencia y la Cultura (2015). La educación para todos, 2000-2015: logros y desafíos. Informe de seguimiento de la EPT en el mundo, 2015. Resumen. Retrieved from http://unesdoc.unesco.org/ images/0023/002325/232565s.pdf.

[17] Organización para la Cooperación y el Desarrollo Económico (2016). Making Development Happen. Avanzando hacia una mejor educación para Perú (vol. 3). Retrieved from http://repositorio.minedu. gob.pe/bitstream/handle/MINEDU/5200/ Avanzando\%20hacia\%20una\%20mejor\%20 educaci\%c3\%b3n\%20para\%20Per\%c3\%ba. pdf? sequence $=1$ \&isAllowed $=y$.

[18] Osorio-Álvarez, M. del C. \& Parra, L. (2016). La satisfacción escolar en los estudiantes del primer año de la carrera de Médico Cirujano. Investigación en Educación Médica, 5(17), 3-10. Retrieved from https://doi.org/10.1016/j. riem.2015.08.002.
[19] Parodi, C. (December 6, 2013). Calidad de la Educación en el Perú. Gestión. Retrieved from https://gestion.pe/blog/ economiaparatodos/2013/12/calidad-de-laeducacion-en-el.html.

[20] Proexpansión (2014). La educación de calidad en Lima. Retrieved from https://proexpansion. com/uploads/report/pdf_file/2/Informe_ educacion_alta_.pdf.

[21] Robalino, M. (July 11, 2016). Perú tiene avances interesantes en el impulso de la calidad educativa. El Peruano. Retrieved from https://elperuano.pe/noticia-"peru-tieneavances-interesantes-el-impulso-de-calidadeducativa"-43625.aspx.

[22] Sánchez, G. (April 11, 2015). ¿Por qué no mejora la educación en América Latina? BBC. Retrieved from https://www.bbc.com/ mundo/noticias/2015/04/150409_economia educacion_en_america_latina_finde_bd.

[23] Saraiva, M. (2008). La calidad y los "clientes" de la enseñanza superior portuguesa. Horizontes Educacionales, 13(2), 41-54. Retrieved from https://dialnet.unirioja.es/descarga/ articulo/3987543.pdf.

[24] Vexler, I. (October 5, 2016). La educación y la competitividad 2016-2017. Correo. Retrieved from https://diariocorreo.pe/ opinion/la-educacion-y-la-competitivid ad-2016-2017-702501.

[25] Villarreal, E. (2014). El reto de la calidad educativa en américa latina. Retrieved from http://www.cacsla.org.mx/informacion-deinteres/el-reto-de-la-calidad-educativa-enamerica-latina. 
